\title{
A LAURENT EXPANSION FOR SOLUTIONS TO ELLIPTIC EQUATIONS
}

\author{
BY
}

REESE HARVEY AND JOHN C. POLKING( $\left.{ }^{1}\right)$

\begin{abstract}
Let $P(\xi)$ be a homogeneous elliptic polynomial of degree $m$. Let $E$ be a fundamental solution for the partial differential operator $P(D)$. Suppose $\Omega$ is a neighborhood of 0 in $\mathbf{R}^{n}$. Suppose $f \in C^{\infty}(\Omega \sim\{0\})$ satisfies $P(D) f=0$ in $\Omega \sim\{0\}$. It is shown that there is a differential operator $H(D)$ (perhaps of infinite order) and a function $g \in C^{\infty}(\Omega)$ satisfying $P(D) g=0$ in $\Omega$, such that $f=H(D) E+g$ in $\Omega \sim\{0\}$. This analog of the Laurent expansion for $f$ is made unique by requiring that the Cauchy principal value of $H(D) E$ be equal to $H(D) E$.
\end{abstract}

Suppose $\Omega$ is an open set in the complex plane containing the origin, and that $f$ is a holomorphic function in $\Omega-\{0\}$. Then $f$ has a Laurent expansion $\Sigma_{-\infty}^{\infty} a_{n} z^{n}$, convergent in some deleted neighborhood of the origin. If the negative part of this Laurent expansion is a finite sum then L. Schwartz ([4] or (II, 3, 25) in [5]) has shown that the Cauchy principal value of $f$ exists in $\mathcal{D}^{\prime}(\Omega)$, and that $\partial / \partial z$ commutes with taking the Cauchy principal value. It is the purpose of this paper to develop a "Laurent expansion" for other elliptic operators.

Let $Q(\xi)=\Sigma_{|a| \leq k} a_{a} \xi^{a}$ be a polynomial with coefficients $a_{a} \in \mathbf{C}$. Here $\alpha=\left(\alpha_{1}, \cdots, \alpha_{n}\right)$ is a multi-index, $|\alpha|=\alpha_{1}+\cdots+a_{n}$, and $\xi^{a}=\xi_{1}^{a_{1}} \cdot \ldots \cdot \xi_{n}^{a_{n}}$. We will write $D^{a}=D_{1}^{a_{1}} \ldots \cdot D_{n}^{a_{n}}$, where $D_{j}=-i\left(\partial / \partial x_{j}\right)$. Then $Q(D)=$ $\Sigma_{|a| \leq k} a_{\alpha} D^{\alpha}$ will be a differential operator: We will be concerned with homogeneous polynomials $Q(\xi)=\Sigma_{|a|=k} a_{a} \xi^{\alpha}$, and we will denote by $\mathcal{P}_{k}$ the space of all homogeneous polynomials of degree $k$. A polynomial $P \in \mathcal{P}_{m}$ is said to be elliptic if $P(\xi) \neq 0$, for all $\xi \in \mathbf{R}^{n} \sim\{0\}$.

Given an open set $\Omega$ contained in $R^{n}$, the space of real-analytic functions on $\Omega$ will be denoted $Q(\Omega)$. Given a compact set $K$ contained in $\mathbf{R}^{n}$ then $\mathbb{Q}(K)$ will denote the space of real-analytic functions on $K$ with the usual (locally convex) inductive limit topology. That is $\mathcal{Q}(K)=\lim _{U \supset K} \mathcal{Q}(U)$, where

Received by the editors August 17, 1972. $45 F 15$.

AMS (MOS) subject classifications (1970). Primary 35C10, 35J30; Secondary 45F10,

Key words and phrases. Elliptic partial differential operator, fundamental solution, Laurent expansion, homogeneous polynomials, solid harmonics, hyperfunction, analytic functional, distribution, Cauchy principal value.

(1) This research was partially sponsored by NSF grants GP 19011 and 33749, respectively. 
the inductive limit is over complex neighborhoods $U$ of $K$ in $\mathbf{C}^{n}$ ( $\mathbf{C}^{n}$ denotes the complexification of $\mathbf{R}^{n}$ ) and $\mathcal{O}(U)$ denotes the space of holomorphic functions on $U$. The space of analytic functionals on $K$ is, by definition $\mathbb{Q}(K)^{\prime}$, the dual space of $\mathcal{A}(K)$. We will let $B(a, r)=\{x:|x-a|<r\}$.

Suppose $P(\xi)$ is a homogeneous elliptic polynomial of degree $m$, and suppose $E$ is a fundamental solution for the differential operator $P(D)$. Suppose $\Omega \subset \mathbf{R}^{n}$ is a neighborhood of the origin. If $f \in \mathscr{T}^{\prime}(\Omega)$ satisfies $P(D) f=0$ in $\Omega \sim\{0\}$, then it is easy to see that there is a polynomial $Q$ and a function $g \epsilon$ $C^{\infty}(\Omega)$, which satisfies $P(D) g=0$ in $\Omega$, such that $f=Q(D) E+g$ in $\Omega \sim\{0\}$ (see the proof of part (2) of the following theorem). Any such equation could be called a Laurent expansion for $f$. The difficulty is that the polynomial $Q$ is not unique; any multiple of $P$ added to $Q$ would do as well since $P(D) E=0$ in $\Omega \sim\{0\}$. The problem then is to put additional conditions on $Q$ so that it will be uniquely determined. In analogy with the situation vis-à-vis the Cauchy Riemann equation as developed by L. Schwartz ([4] or (II, 3, 25) in [5]), we demand that the Cauchy principal value of $Q(D) E$ exist and be equal to $Q(D) E$.

Definition 1. Suppose $P(\xi)$ is a homogeneous elliptic polynomial of degree m. Let $\mathcal{H}_{k}$ denote the space of polynomials $H \in \mathcal{P}_{k}$ for which

$$
\int_{|\xi|=1} \frac{H(\xi) \xi^{a}}{P(\xi)} d \sigma(\xi)=0
$$

for all multi-indices $\alpha$ with $|\alpha|=k-m$. If $k-m<0$, define $\mathcal{H}_{k}=\mathscr{P}_{k}$. Set $\mathcal{H}=\Sigma_{k=0}^{\infty} \oplus \mathcal{H}_{k}$.

Remark. If $H \in \mathcal{H}_{k}$, then $\int|\xi|=1 H(\xi) \xi^{\alpha} / P(\xi) d \sigma(\xi)=0$ for all $|\alpha| \leq k-m$. For $|a|=k-m-2 j+1$, this follows since the integrand is odd, and for $|\alpha|=k-m-2 j$, we have $\xi^{a}=|\xi|^{2 j} \xi^{a}$ on the domain of the integral, and $|\xi|^{2 j} \xi^{a}$ is a polynomial of degree $k-m$.

Lemma 1. There is a direct sum decomposition

$$
\mathcal{P}_{k}=P \mathcal{P}_{k-m} \oplus \mathcal{H}_{k} \text {. }
$$

In fact $\mathcal{H}_{k}$ and $P \mathcal{P}_{k-m}$ are ortbogonal complements with respect to the inner product

$$
\langle Q, S\rangle=\int_{|\xi|=1} \frac{Q(\xi) \overline{S(}(\xi)}{|P(\xi)|^{2}} d \sigma(\xi)
$$

defined on $\mathscr{P}_{k}$.

Proof. By definition $\mathcal{H}_{k}$ is the orthogonal complement of $P \mathcal{P}_{k-m}$.

Of course the direct sum decomposition $\mathfrak{P}_{k}=P \mathcal{P}_{k-m} \oplus \mathcal{H}_{k}$ does not uniquely determine $H_{k}$. 
Definition 2. Let $\Omega$ be an open set in $\mathbf{R}^{n}$, which contains the origin. Let $\chi_{\epsilon}$ denote the characteristic function of the set $\{x:|x| \geq \epsilon\}$. Suppose $f$ is a locally integrable function in $\Omega \sim\{0\}$. Then $\chi_{\epsilon} f \in \mathscr{D}^{\prime}(\Omega)$ for all $\epsilon>0$. If $\lim _{\epsilon \rightarrow 0} \chi_{\epsilon} f$ exists weakly in $\mathscr{T}^{\prime}(\Omega)$, the limit is called the Caucby principal value of $f$ and is denoted by $\mathrm{pv} f$.

Remark. A standard argument shows that if $\lim _{\epsilon \rightarrow 0} \chi_{\epsilon} f$ exists in the weak topology on $\mathscr{D}^{\prime}(\Omega)$ then $\left\{\chi_{\epsilon} f: 0<\epsilon \leq \delta\right\}$ is relatively weakly compact and hence that $\lim _{\epsilon \rightarrow 0} X_{\epsilon} f$ exists in the strong topology on $\mathscr{D}^{\prime}(\Omega)$ (see Schwartz $[5$, p. 74]).

Examples. In $\mathbf{R}^{1}$, the Cauchy principal value of $f(t)=t^{-1}$ exists while that of $g(t)=|t|^{-1}$ does not.

Theorem. Suppose $P(\xi)$ is a bomogeneous, elliptic polynomial of degree $m$, and $\mathcal{H}_{k}$ is as defined above. Let $E(x)$ be a fundamental solution for $P(D)$.

(1) The Caucby principal value of $Q(D) E$ exists (strongly) in $\mathscr{D}^{\prime}\left(\mathbf{R}^{n}\right)$ for all polynomials $Q$, and pv $Q(D) E=Q(D) E$ if and only if $Q \in \mathcal{H}$.

(2) Suppose $f \in \mathscr{D}^{\prime}(\Omega)$ satisfies $P(D) f=0$ in $\Omega-\{0\}$. There exists a unique $H \in \mathcal{H}$ and a $g \in C^{\infty}(\Omega)$ satisfying $P(D) g=0$ in $\Omega$, such that $f=$ $H(D) E+g$ in $\Omega-\{0\}$. Therefore by (1) the Caucby principal value of $\left.\left.f\right|_{\Omega-\{0}\right\}$ exists (strongly) in $\mathfrak{D}^{\prime}(\Omega)$. Moreover if $f(x)=o\left(|x|^{m-n-k-1}\right)$ and $k \geq m-n$ then $\operatorname{deg} H \leq k$.

(3) Suppose $f \in \mathbb{P}(\Omega \sim\{0\})$ satisfies $P(D) f=0$ in $\Omega \sim\{0\}$. Then there exists a unique $H_{k} \in \mathcal{H}_{k}$ for $k=0,1, \cdots$ and a unique $g \in \mathbb{T}(\Omega)$ satisfying $P(D) g=0$ in $\Omega$ such that $f(x)=\sum_{k=0}^{\infty} H_{k}(D) E(x)+g(x)$ in $\Omega \sim\{0\}$. The sum converges uniformly on compact subsets of $\Omega \sim\{0\}$. Moreover, the Caucby principal value of $f$ exists in the sense that $\chi_{\epsilon} f-\Sigma H_{k}(D) E-g$ converges to zero in $\mathbb{A}(B(0,1))^{\prime}$.

Remark. Suppose $P(x, D)$ is an elliptic differential operator with infinitely differentiable coefficients in $\Omega$. Suppose $f \in \mathscr{I}^{\prime}(\Omega)$ satisfies $P(x, D) f=0$ in $\Omega \sim\{0\}$. It remains true that the Cauchy principal value of $f$ exists in $\mathscr{D}^{\prime}(\Omega)$; however, it is not as easy to identify $\mathrm{pv} f$ in the general case.

Examples. (1) For the Cauchy Riemann operator $\partial / \partial \bar{z}=1 / 2(\partial / \partial x+i \partial / \partial y)$, it: is convenient to use complex coordinates $(z, \bar{z})$ instead of the real coordinates $(x, y)$. The elliptic polynomial is $P(z, \bar{z})=z / 2$. A fundamental solution is $E(z, \bar{z})=(\pi z)^{-1}$. It is easy to verify that the only polynomial in $\mathcal{H}_{k}$ is $H_{k}(z, \bar{z})=\bar{z}^{k}$; the corresponding operator is (up to a multiplicative constant) $(\partial / \partial z)^{k}$. Thus the expansion of the theorem is the Laurent expansion and the theorem contains the results of Schwartz ([4], [5]).

(2) Let $P(\xi)=|\xi|^{2}$. Then $P(D)=-\Delta=-\left[\left(\partial / \partial x_{1}\right)^{2}+\cdots+\left(\partial / \partial x_{n}\right)^{2}\right]$. In this case $\mathcal{H}_{k}$ is the space of all homogeneous harmonic polynomials of degree $k$, 
i.e., the solid harmonics of degree $k$. To see this it suffices to show that the solid harmonics are contained in $\mathcal{H}_{k}$, since the two spaces have the same dimension. But now if $Q$ is a polynomial of degree $k-2$, we have that $Q(\xi)=$ $\Sigma|\xi|^{2 \nu} R_{k-2-2 \nu}(\xi)$ where $R_{j}$ is a solid harmonic of degree $j$ (see [1]). Then if $H$ is a solid harmonic of degree $k$, we have $\langle H, Q\rangle=\Sigma\left\langle H, R_{k-2-2 \nu}\right\rangle=0$ since any two solid harmonics of different degree are orthogonal; consequently $H \in \mathcal{H}_{k}$.

Restricting ourselves to $\mathbf{R}^{n}$ with $n \geq 3$, we choose $E(x)=\left[\omega_{n}(n-2)\right]^{-1}|x|^{2-n}$ for a fundamental solution, where $\omega_{n}$ is the surface area of the $(n-1)$-sphere in $\mathbf{R}^{n}$. It follows from Hecke's identity that if $H_{k}$ is a solid harmonic of degree $k$, then $H_{k}(D) E(x)=c_{k n} H_{k}(x)|x|^{2-n-2 k}$, where $c_{k n}$ is a constant depending only on $k$ and $n$. Thus if $f \in \mathbb{Q}(\Omega-\{0\})$ satisfies $\Delta f=0$ in $\Omega \sim\{0\}$, there are unique solid harmonics $H_{k}$ and $Q_{k}$ of degree $k$ such that

$$
f(x)=\sum_{k=0}^{\infty} H_{k}(x)|x|^{2-n-2 k}+\sum_{k=0}^{\infty} Q_{k}(x) .
$$

This is the classical expansion of $f$ in terms of solid harmonics.

(3) If $P(D)=\Delta^{m}$ then it is easily verified that $\mathcal{H}_{k}=\left\{Q \in \mathcal{P}_{k}: \Delta^{m} Q=0\right\}$.

Proof. (1) Suppose $Q \in \mathcal{P}_{k}$. By the lemma $Q=R P+H$ where $H \in \mathcal{H}_{k}$. Then $Q(D) E=H(D) E$ in $\mathbf{R}^{n} \sim\{0\}$, so it suffices to show that the Cauchy principle value of $H(D) E$ exists and equals $H(D) E$.

If $E_{1}$ and $E_{2}$ are fundamental solutions for $P(D)$, then $E_{1}-E_{2} \in C^{\infty}\left(\mathbf{R}^{n}\right)$. Consequently the result is independent of the particular fundamental solution. We let $E$ denote the fundamental solution constructed by John [2]. That is, $E(x)$ is real analytic in $\mathbf{R}^{n} \sim\{0\}, E(x)$ is positively homogeneous of degree $m-n$ if $m<n$, and $E(x)=E_{0}(x)+E_{1}(x) \log |x|$ if $m \geq n$, where $E_{0}(x)$ is homogeneous of degree $m-n$ and $E_{1}(x)$ is a homogeneous polynomial of degree $m-n$.

If $k<m$, then $H(D) E \in L_{\text {loc }}^{1}\left(\mathbf{R}^{n}\right)$ so the Cauchy principal value of $H(D) E$ exists and equals $H(D) E$ for all $H \in \mathcal{H}_{k}=\mathcal{P}_{k}$.

If $k \geq m$, then $H(D) E$ is a homogeneous distribution in $\mathbf{R}^{n}$ of degree $m-$ $n-k_{0}$ We will show that $\left(1-\chi_{\epsilon}^{\prime}\right) H(D) E$ converges to zero strongly in $\mathscr{D}^{\prime}\left(\mathbf{R}^{n}\right)$. Let $\psi_{\epsilon}=1-\chi_{\epsilon}$. For $\phi \in C_{0}^{\infty}\left(\mathbf{R}^{n}\right)$, we set $\phi(x)=p(x)+r(x)$ by Taylor's theorem, where $p$ is a polynomial of degree $k-m$ and $\left|D^{\alpha}{ }_{r}(x)\right| \leq C_{\alpha}|x|^{k-m-|\alpha|+1}$ for all $\alpha$, and for all $|x| \leq 2$. If we set $r_{\epsilon}(x)=\epsilon^{m-k} r(\epsilon x)$, then $\left|D^{\alpha} r_{\epsilon}(x)\right| \leq C_{\alpha} \epsilon$ for $|x| \leq 2$. Therefore $r_{\epsilon}$ converges to zero in the space $C^{\infty}(\{x:|x|<2\})$. Using the homogeneity of $H(D) E$ we have $\left(\psi_{\epsilon} H(D) E, r\right)=\left(\psi_{1} H(D) E, r_{\epsilon}\right)$. Since $r_{\epsilon} \rightarrow 0$ this proves that $\left(\psi_{\epsilon} H(D) E, r\right)$ converges to zero.

To complete the proof of (1) it suffices to show that $\left(\psi H(D) E, x^{\alpha}\right)=0$ for $|a| \leq k-m$, where $\psi$ is a radial function with compact support, and is equal to one near the origin. It clearly suffices to assume that $\psi \in C_{0}^{\infty}\left(\mathbf{R}^{n}\right)$. We notice 
that since $H(D) E$ is a homogeneous distribution of degree $m-n-k$, its Fourier transform $H(\xi) \hat{E}(\xi)$ is homogeneous of degree $k-m \geq 0$. Thus $H(\xi) \hat{E}(\xi)$ is determined by its values in $\mathbf{R}^{n} \sim\{0\}$. Since $\hat{E}(\xi)=P(\xi)^{-1}$ for $\xi \neq 0$ we have $(H(D) E)^{\wedge}(\xi)=H(\xi) / P(\xi)$. Consequently by Parseval's formula

$$
\begin{aligned}
\left(\psi H(D) E, x^{\alpha}\right) & =\left(H(D) E, x^{\alpha} \psi\right)=\left(H / P, D^{\alpha} \hat{\psi}\right) \\
& =\int \frac{H(\xi) \overline{D^{\alpha} \hat{\psi}(\xi)}}{P(\xi)} d \xi .
\end{aligned}
$$

Since $\psi \in C_{0}^{\infty}\left(\mathbf{R}^{n}\right)$ is radial, its Fourier transform $\hat{\psi}$ is an infinitely differentiable, even, radial function. Therefore we can write $\hat{\psi}(\xi)=f\left(|\xi|^{2}\right)$. It then follows easily by induction on $|a|$ that

$$
D^{\alpha} \hat{\psi}(\xi)=\sum_{j=0}^{|\alpha|-1} f^{(|\alpha|-j)}\left(|\xi|^{2}\right) Q_{j}^{\alpha}(\xi)
$$

where $Q_{j}^{a}$ is a homogeneous polynomial of degree $|\alpha|-2 j$ if $|\alpha| \geq 2 j$, and is identically zero otherwise. Since $|\alpha| \leq k-m$ and $H \in \mathcal{H}_{k}$ (see Definition 1 and remark afterward), we have that $\int_{|\xi|=1} H(\xi) Q_{j}^{a}(\xi) / P(\xi) d \sigma(\xi)=0$. Thus $\left(\psi H(D) E, x^{\alpha}\right)=0$ for $|\alpha| \leq k-m$.

(2) By hypothesis $P(D) f$ is supported at the origin, so there is a polynomial $Q$ such that $P(D) f=Q(D) \delta$, where $\delta$ is the Dirac measure at the origin. Let $f_{1}=P(D) f * E=Q(D) E$. Then $P(D) f_{1}=P(D) f$ so if $g=f-f_{1}$ we have $P(D) g=0$ in $\Omega$. Furthermore $f=Q(D) E+g$ in $\Omega \sim\{0\}$. By Lemma 1 there is a unique $H \in \mathcal{H}$ such that $Q=R P+H$. Therefore $Q(D) E=R(D) \delta+H(D) E$ and hence $f=H(D) E+g$ on $\Omega \sim\{0\}$. This proves the first part of (2). The fact that the Cauchy principal value of $f$ exists now follows from (1).

Suppose now that $f(x)=o\left(|x|^{m-n-k-1}\right)$ and $k \geq m-n$. Let $H=\sum_{j=0}^{l} H_{j}$, where $H_{j} \in \mathcal{H}_{j}$ and $H_{l} \neq 0$. Suppose $l>k$. Then $H_{l}(D) E(x)=f(x)-g(x)-$ $\sum_{j=0}^{l-1} H_{j}(D) E(x)=o\left(|x|^{m-n-l}\right)$. Since $H_{l}(D) E$ is homogeneous of degree $m-n-l$, this implies that $H_{l}(D) E=0$ in $\mathbf{R}^{n} \sim\{0\}$. Then by $(1), H_{l}(D) E=0$ in $\mathbf{R}^{n}$. Applying $P(D)$ we get $H_{l}(D) \delta=0$, which means that $H_{l}=0$.

In dealing with infinite expansions, convergence problems arise. To handle them we use the following result. For $Q(\xi)=\Sigma_{|a|=k} a_{a} \xi^{a} \in \mathcal{P}_{k}$, let $|Q|=$ $\sup _{|a|=k}\left|a_{a}\right|$, and let $\|Q\|^{2}=\int_{|\xi|=1}|Q(\xi)|^{2} d \xi$.

Lemma 2. There is a constant $C$ depending only on $n$ such that $C^{-k}|Q| \leq$ $\|Q\| \leq C^{k}|Q|$ for all $Q \in \mathcal{P}_{k}$.

Proof. Clearly $\|Q\|^{2} \leq \omega_{n}|Q|^{2} \operatorname{dim} \mathscr{P}_{k}$. Since $\operatorname{dim} \mathscr{P}_{k}=\left(\begin{array}{c}k+n-1 \\ n-1\end{array}\right) \leq n^{k}$, the second inequality follows. 
To prove the first inequality we refer to a result in $[1$, p. 33] which implies that there is a constant $C$ depending only on $n$ such that if $H(\xi)=$ $\sum_{|a|=k} b_{a} \xi^{a}$ is a solid harmonic of degree $k$, then $a !\left|a_{a}\right| \leq C k^{n / 2+k-1}\|H\|$. Using the formula $n^{k}=\Sigma_{|a|=k} k ! / \alpha$ !, the fact that $k \leq 2^{k}$, and Stirling's formula, we have the first inequality of the lemma for harmonic polynomials.

For general $Q \in \mathcal{P}_{k}$ there are harmonic polynomials $H_{k-2 \nu}$ of degree $k-2 \nu$, such that $Q(\xi)=\Sigma|\xi|^{2 \nu} H_{k-2 \nu}(\xi) .|\xi|^{2 \nu}=\Sigma|a|=\nu(\nu ! / \alpha !) \xi^{\alpha} ;$ this expansion has fewer than $n^{\nu}$ terms and each coefficient is smaller than $n^{\nu}$. Consequently

$$
\begin{aligned}
|Q| \leq \sum n^{2 \nu}\left|H_{k-2 \nu}\right| & \leq \sum n^{2 \nu} C^{k-2 \nu}\left\|H_{k-2 \nu}\right\| \\
& \leq(n C)^{k} \sum\left\|H_{k-2 \nu}\right\| \leq(n C)^{k} k^{1 / 2}\left(\sum\left\|H_{k-2 \nu}\right\|^{2}\right)^{1 / 2} .
\end{aligned}
$$

Since $\|Q\|^{2}=\Sigma\left\|H_{k-2 \nu}\right\|^{2}$, the proof of the lemma is complete.

(3) The function $f$ has a hyperfunction extension $f_{1}$ (see Sato [3]). $P(D) f_{1}$ is a hyperfunction supported at the origin and consequently is of the form $\Sigma_{k=0}^{\infty} Q_{k}(D) \delta$, where $Q_{k} \in \mathcal{P}_{k}$ and for all $\epsilon>0,\left|Q_{k}\right|=o\left(\epsilon^{k} / k !\right)$ as $k \rightarrow \infty$. By Lemma 1 , for each $k$ there are $R_{k} \in \mathscr{P}_{k-m}$ and $H_{k} \in \mathcal{H}_{k}$ such that $Q_{k}=P R_{k}$ $+H_{k}$. Furthermore, since the decomposition in Lemma 1 is orthogonal we have $\left\|H_{k} / P\right\| \leq\left\|Q_{k} / P\right\|$. Since $|P(\xi)|$ is bounded above and bounded away from zero on the unit sphere, this inequality together with Lemma 2 implies that $\left|H_{k}\right|=$ $o\left(\epsilon^{k} / k !\right)$ as $k \rightarrow \infty$ for all $\epsilon>0$, and the same for $R_{k}$. Thus if we let $Q=\Sigma_{k=0}^{\infty} Q_{k}, R=\sum_{k=0}^{\infty} R_{k}, H=\sum_{k=0}^{\infty} H_{k}, Q(D) \delta, R(D) \delta$, and $H(D) \delta$ are all hyperfunctions supported at the origin and we have $Q(D) \delta=R(D) P(D) \delta+H(D) \delta$. Let $f_{2}=P(D) * E=R(D) \delta+H(D) E$, and let $g=f_{1}-f_{2}$. Then $P(D) g=0$ in $\Omega$ since $P(D) f_{2}=P(D) f_{1}$. Furthermore $f=H(D) E+g$ in $\Omega \sim\{0\}$.

To show that the Cauchy principal value exists, it clearly suffices to show that $\psi_{\epsilon} H(D) E \rightarrow 0$ in $\overline{\mathbb{Q}^{\prime}(B(0,1))}$. Let $\phi \in \overline{\mathbb{Q}(B(0,1))}$. Then $\phi(x)=\Sigma a_{a} x^{a}$ converges for $|x|<\delta$ for some $\delta>0$. This implies that $\left|a_{a}\right| \leq M \delta^{-}|a|$. If $\epsilon<\delta$ we have

$$
\left(\psi_{\epsilon} H(D) E, \phi\right)=\sum_{k=0}^{\infty} \sum_{a} a_{a}\left(\psi_{\epsilon} H_{k}(D) E, x^{a}\right) .
$$

In the proof of $(1)$ we showed that $\left(\psi_{\epsilon} H_{k}(D) E, x^{a}\right)=0$ if $|\alpha| \leq k-m$. To handle the remaining terms we need estimates on $H_{k}(D) E . E$ is analytic in $\mathbf{R}^{n} \sim\{0\}$. Consequently, there is a constant $M$ such that $\left|D^{\beta} E(x)\right| \leq M^{k+1} k !$ for $|x|=1$ and for all $|\beta|=k$. If $k>m-n, D^{\beta} E$ is homogeneous of degree $m-n-k$. Consequently $\left|D^{\beta} E(x)\right| \leq M^{k+1} k !|x|^{m-n-k}$ for all $|\beta|=k>m-n$. It follows that $\left|H_{k}(D) E(x)\right| \leq\left|H_{k}\right| M^{k+1} k !|x|^{m-n-k}$, and, from the estimates on $\left|H_{k}\right|$, that for 
every $\eta>0$, there is a constant $C_{\eta}$ such that $\left|H_{k}(D) E(x)\right| \leq C_{\eta} \eta^{k}|x|^{m-n-k}$ for all $x \neq 0$, provided that $k>m-n$.

By (1) we have

$$
\left(\psi_{\epsilon} H_{k}(D) E, x^{\alpha}\right)=\lim _{\eta \rightarrow 0} \int_{\eta \leq|x| \leq \epsilon} H_{k}(D) E(x) x^{\alpha} d x=\int_{|x| \leq \epsilon} H_{k}(D) E(x) x^{\alpha} d x
$$

if $|\alpha|>k-m$. By the estimates on $H_{k}(D) E$, we have $\left|\left(\psi_{\epsilon} H_{k}(D) E, x^{\alpha}\right)\right| \leq$ $C_{\eta} \eta^{k} \epsilon^{|\alpha|+m-k}$ if $|\alpha|>k-m$. Thus if we choose $\eta<\delta / n$ we have

$$
\left|\left(\psi_{\epsilon} H(D) E, \phi\right)\right| \leq \sum_{k=0}^{\infty} \sum_{|\alpha|>k-m}\left|a_{a}\right|\left|\left(\psi_{\epsilon} H_{k}(D) E, x^{\infty}\right)\right|=O(\epsilon) .
$$

(The convergence is uniform in $\phi$ satisfying $\left|a_{a}\right| \leq M \delta^{-|a|}$ with $M$ and $\delta$ fixed, and hence in the strong topology on $\left(\mathcal{P}(\overline{B(0,1)})^{\prime}\right.$.)

Since $P(D) H(D) E=0$ in $\mathbf{R}^{n} \sim\{0\}$, it follows from a standard argument that the sum converges uniformly on compact subsets of $\mathbf{R}^{n} \sim\{0\}$ (in fact it follows that $H(D) E$ converges in $\mathbb{P}(K)$ for all compact sets $\left.K \subset \mathbf{R}^{n} \sim\{0\}\right)$. This also follows easily from the fact proved above, that for every $\eta>0$, there is a constant $C_{\eta}$ such that $\left|H_{k}(D) E(x)\right| \leq C_{\eta} \eta^{k}|x|^{m-n-k}$ for all $x \neq 0$ provided that $k>m-n$.

\section{BIBLIOGRAPHY}

1. A.-P. Calderón, Singular integrals and their applications to hyperbolic differential equation, Curos y Seminarios de Matematica, fasc. 3, Universidad de Buenos Aires, Buenos Aires, 1960。(Spanish) MR 23 \#A1156.

2. F。 John, Plane waves and spherical means applied to partial differential equations, Interscience, New York, 1955. MR 17, 746。

3. M. Sato, Theory of hyperfunctions. II, J. Fac. Sci. Univ. Tokyo Sect。 I 8 (1960), 387-437. MR 24 \# A2237.

4. L.Schwartz, Courant associé à une forme différentielle méromorphe sur une variété analytique complexe. Géométrie différentielle, Colloq. Internationaux du Centre National de la Recherche Scientifique, Strasbourg, 1953. Centre National de la Recherche Scientifique, Paris, 1953, pp。185-195. MR 16, 518.

5. - Théorie des distributions, Publ. Inst. Math. Univ. Strasbourg, no.9-10, Hermann, Paris, 1966. MR $35 \# 730$. 